\title{
The interplay between $d$-wave superconductivity and antiferromagnetic fluctuations: a quantum Monte Carlo study
}

\author{
F.F. Assaad \\ Institut für Theoretische Physik III, \\ Universität Stuttgart, Pfaffenwaldring 57, D-70550 Stuttgart, Germany.
}

We consider the repulsive Hubbard model on a square lattice with an additional term, $W$, which depends upon the square of a single-particle nearest-neighbor hopping. At halfband filling, constant $W$, we show that enhancing $U / t$ drives the system from a $d$-wave superconductor to an antiferromagnetic Mott insulator. At zero temperature in the superconducting phase, spin-spin correlations follow a powerlaw: $e^{-i \vec{r} \cdot \vec{Q}}|\vec{r}|^{-\alpha}$. Here $\vec{Q}=(\pi, \pi)$ and $\alpha$ is in the range $1<\alpha<2$ and depends upon the coupling constants $W$ and $U$. This results is reached on the basis of large scale quantum MonteCarlo simulations on lattices up to $24 \times 24$, and is shown to be independent on the choice of the boundary conditions. We define a pairing (magnetic) scale by the temperature below which short range $d$-wave pairing correlations (antiferromagnetic fluctuations) start growing. With finite temperature quantum Monte Carlo simulations, we demonstrate that both scales are identical over a large energy range. Those results show the extreme compatibility and close interplay of antiferromagnetic fluctuations and $d$-wave superconductivity.

The understanding of the interplay between $d$-wave superconductivity and antiferromagnetism is a central issue for the understanding of the phase diagram of High- $T_{c}$ superconductors [1]. The aim of this work is to further study a model which shows a quantum transition between a $d$-wave superconductor and an antiferromagnetic Mott insulator. It thus enables us to address the above question. The model we consider, has been introduced in Ref. [2]. It is defined by:

$H=-\frac{t}{2} \sum_{\vec{i}} K_{\vec{i}}-W \sum_{\vec{i}} K_{\vec{i}}^{2}+U \sum_{\vec{i}}\left(n_{\vec{i}, \uparrow}-\frac{1}{2}\right)\left(n_{\vec{i}, \downarrow}-\frac{1}{2}\right)$

with the hopping kinetic energy

$$
K_{\vec{i}}=\sum_{\sigma, \vec{\delta}}\left(c_{\vec{i}, \sigma}^{\dagger} c_{\vec{i}+\vec{\delta}, \sigma}+c_{\vec{i}+\vec{\delta}, \sigma}^{\dagger} c_{\vec{i}, \sigma}\right) .
$$

Here, $W \geq 0, \vec{\delta}= \pm \vec{a}_{x}, \pm \vec{a}_{y}$, and $n_{\vec{i}, \sigma}=c_{\vec{i}, \sigma}^{\dagger} c_{\vec{i}, \sigma}$ where $c_{\vec{i}, \sigma}^{\dagger}\left(c_{\vec{i}, \sigma}\right)$ creates (annihilates) an electron on site $\vec{i}$ with z-component of spin $\sigma$. We impose twisted boundary conditions:

$$
c_{\vec{i}+L \vec{a}_{x}, \sigma}=\exp \left(2 \pi i \Phi / \Phi_{0}\right) c_{\vec{i}, \sigma}, \quad c_{\vec{i}+L \vec{a}_{y}, \sigma}=c_{\vec{i}, \sigma},
$$

with $\Phi_{0}=h c / e$ the flux quanta and $L$ the linear length of the square lattice. The boundary conditions given by Eq. (3) account for a magnetic flux threading a torus on which the lattice is wrapped. One major advantage of the above model consists in the fact that at half-band filling, the sign problem in the quantum Monte-Carlo (QMC) method may be avoided. This statement is valid even for the above boundary conditions. Hereafter, we will only consider the half-filled case.

Previously we have considered the model of Eq. (11) at fixed values of $U$ and as a function of $W$. At $U / t=4$ we have shown that the quantum transition between the Mott insulator and $d$-wave superconductor occurs at $W_{c} / t \sim 0.3$ [2]. We have equally considered the model at finite doping and given numerical evidence of the occurrence of a doping induced quantum transition between the Mott insulator and $d$-wave superconductor [3]. Here, we fix $W / t=0.35$ and vary $U / t$ for the half-filled case. The advantage of this choice of parameters is that it provides us with a large parameter range in the superconducting phase where QMC simulations are extremely precise, thus allowing us to reach large lattices. This allows us to reliably study the nature of the spin degrees of freedom in the superconducting state. We have used two QMC algorithms. i) The Projector QMC algorithm which produces zero temperature results in a canonical ensemble [4,5] and ii) the finite temperature grand canonical QMC method [6,7]. The application of those algorithms for the above model has been discussed in reference [2]. Both algorithms, generate a systematic error proportional to $(\Delta \tau)^{2}$ where $\Delta \tau$ denotes the imaginary time step. To determine the exponent of the spin-spin correlations we have extrapolated $\Delta \tau$ to zero.

We first concentrate of the charge degrees of freedom at zero temperature. To distinguish between a superconductor and insulator, we compute the ground state energy as a function of the twist in the boundary condition: $E_{0}(\Phi)$. For an insulator, the wave function is localized and hence, an exponential decay of $\Delta E_{0}(\Phi) \equiv$ $E_{0}(\Phi)-E_{0}\left(\Phi_{0} / 2\right)$ as a function of lattice size is expected [8]. In the Hartree-Fock spin density wave (SDW) approximation for the half-filled Hubbard model, one obtains $\Delta E_{0}(\Phi)=\alpha(\Phi) L \exp (-L / \xi)$ where $\xi$ is the localization length of the wave function. On the other hand, for a superconductor, $\Delta E_{0}(\Phi)$ shows anomalous flux quantization: $\Delta E_{0}(\Phi)$ is a periodic function of $\Phi$ with period $\Phi_{0} / 2$ and a non vanishing energy barrier is to be found between the flux minima 9 11] so that 
$\Delta E_{0}\left(\Phi_{0} / 4\right)$ remains finite as $L \rightarrow \infty$. Fig. (11) plots $\Delta E_{0}(\Phi / 4)$ versus $1 / L$ at $W / t=0.35$ and for various values of $U / t$. At values of $U / t \leq 4$, the data is consistent with a $1 / L$ form and scales to a finite value. At $U / t=5$, the data may be fitted to the above SDW form. Thus, for values of $W / t=0.35$, the data is consistent with the occurrence of a quantum transition between a superconductor and a Mott insulator at $4<U_{c} / t<5$.

In order to determine the symmetry of the superconducting state, we have computed equal time pairing correlations in the extended $s$-wave and $d_{x^{2}-y^{2}}$ channels:

$$
P_{d, s}(\vec{r})=\left\langle\Delta_{d, s}^{\dagger}(\vec{r}) \Delta_{d, s}(\overrightarrow{0})\right\rangle
$$

with

$$
\Delta_{d, s}^{\dagger}(\vec{r})=\sum_{\sigma, \vec{\delta}} f_{d, s}(\vec{\delta}) \sigma c_{\vec{r}, \sigma}^{\dagger} c_{\vec{r}+\vec{\delta},-\sigma}^{\dagger} .
$$

Here, $f_{s}(\vec{\delta})=1$ and $f_{d}(\vec{\delta})=1(-1)$ for $\vec{\delta}= \pm \overrightarrow{a_{x}}\left( \pm \overrightarrow{a_{y}}\right)$. The vertex contribution to the above quantity is given by:

$$
\begin{aligned}
& P_{d, s}^{v}(\vec{r})=P_{d, s}(\vec{r})-\sum_{\sigma, \vec{\delta}, \vec{\delta}^{\prime}} f_{d, s}(\vec{\delta}) f_{d, s}\left(\vec{\delta}^{\prime}\right) \\
& \left(\left\langle c_{\vec{r}, \sigma}^{\dagger} c_{\vec{\delta}^{\prime}, \sigma}\right\rangle\left\langle c_{\vec{r}+\vec{\delta},-\sigma}^{\dagger} c_{\overrightarrow{0},-\sigma}\right\rangle+\left\langle c_{\vec{r}, \sigma}^{\dagger} c_{\overrightarrow{0}, \sigma}\right\rangle\left\langle c_{\vec{r}+\vec{\delta},-\sigma}^{\dagger} c_{\vec{\delta}^{\prime},-\sigma}\right\rangle\right) .
\end{aligned}
$$

Per definition, $P_{d, s}^{v}(\vec{r}) \equiv 0$ in the absence of interactions. Fig. 2 plots $P_{d}^{v}(\vec{r})$ along the diagonal of the lattice. We consider lattices ranging up to $L=24$. For a fixed lattice size, one notices a plateau structure as a function of distance. The extrapolation of this plateau value to the thermodynamic limit is hard and the above criterion of flux quantization, proves to be a more efficient method to conclude superconductivity [12. In comparison to the $d$-wave signal, the extended $s$-wave signal at large distances (data not shown) may not be distinguished from zero within our accuracy. The extended $s$-wave pair-field correlation dominate at short distances. The data confirming this statement may be found in the table.

The added $W$-term, contains no processes which explicitly favor $d$-wave superconductivity. On the contrary, one would expect the $W$-term to favor extended $s$-wave symmetry, and this shows up on short length scales. The fact that $d$-wave symmetry dominates at long-range is a result of the underlying magnetic structure.

To study the spin degrees of freedom, we have computed equal time spin-spin correlations

$$
S(\vec{r})=\frac{4}{3}\langle\vec{S}(\vec{r}) \vec{S}(\overrightarrow{0})\rangle
$$

where $\vec{S}(\vec{r})$ denotes the spin operator on site $\vec{r}$. Fig. 3 , plots $S(L / 2, L / 2)$ versus $1 / L$ where $L$ corresponds to the linear size of the lattice. We consider periodic boundary conditions, and various values of $U / t$. W/t is constant and set to $W / t=0.35$. For values of $U / t \leq 4$ the data is consistent with a powerlaw decay:

$$
\begin{aligned}
& S(L / 2, L / 2) \sim L^{-1.49} \text { for } U / t=1 \\
& S(L / 2, L / 2) \sim L^{-1.32} \text { for } U / t=2 \\
& S(L / 2, L / 2) \sim L^{-1.17} \text { for } U / t=3 \\
& S(L / 2, L / 2) \sim L^{-1.01} \text { for } U / t=4 .
\end{aligned}
$$

At $U / t=5$ extrapolation of the data leads to a finite staggered moment, and thus the presence of antiferromagnetic long-range order. The statement in Eq. (8) is surprising and deserves confirmation. To cross-check the validity of the above equation, we demonstrate numerically that it is independent on the choice of the boundary condition. Fig. 1 plots $S(L / 2, L / 2)$ at $U / t=1$ and $U / t=2$ for $W / t=0.35$. At $\Phi=0$, the solid lines corresponds to least square fits to the form $c L^{-\alpha}$. We thus determine the exponent $\alpha$. The data at $\Phi=\Phi_{0} / 2$ is consistent at large distances with the form $\tilde{c} L^{-\alpha}$ thus showing that the exponent $\alpha$ is independent on the choice of the boundary. For the $\Phi=\Phi_{0} / 2$ simulations we were able to reach lattices up to $24 \times 24$. At $U / t=2$ and for the boundary conditions set by $\Phi=\Phi_{0} / 2, P_{d}^{v}(L / 2, L / 2)$ (see Fig. 2) decays slower than $S(L / 2, L / 2)$ (see Fig. 1) thus confirming that the pairing correlations are dominant [13].

Having put Eq. (8) on a numerically firm basis, we now argue why it is a surprising result. In two dimensions, correlation functions which decay slower than $1 / r^{2}$ lead to divergences in Fourier space. The Fourier transform of the spin-spin correlations at $U / t=2$ is plotted in Fig. 5. One sees a systematic increase of $S(\vec{Q}=(\pi, \pi))$ as a function of system size [14]. In contrast, a mean field $d$ wave BCS calculation, yields spin-spin correlations which decays as a powerlaw with $\alpha_{B C S}=3.5$. This meanfield result leads to a finite $S(\vec{Q})$ in the thermodynamic limit. Another surprise comes from the dependence of the exponent $\alpha$ on the coupling constants, $U$ and $W$. This is a feature which occurs in one dimensional quantum systems such as the $t-J$ or Hubbard models.

We now consider the model at finite temperatures with the use of the grand-canonical QMC algorithm. In Fig. 6 (a) we plot $\mathrm{S}(\mathrm{Q})$ for values of $U / t$ ranging from $U / t=0$ to $U / t=8$. We define the magnetic scale, $T_{J}$, as the temperature scale where $S(Q)$ starts growing. In Figs. 6 (b) and (c) we consider the vertex contribution to the $d$-wave paring correlations at distance $\vec{R}=(L / 2, L / 2)$ : $P_{d}^{v}(L / 2, L / 2)$. Here, we consider an $L=10$ lattice. At this distance the $s$-wave pairing correlations are negligible. We define the $d$-wave pairing scale, $T_{p}^{d}$, by the temperature scale at which $P_{d}^{v}(L / 2, L / 2)$ starts growing. From the data, we conclude that

$$
T_{p}^{d} \equiv T_{J}
$$

That is, antiferromagnetic fluctuations as well as $d$-wave pairing fluctuations occur hand in hand at the same en- 
ergy scale. If $U / t>U_{c} / t\left(U / t<U_{c} / t\right)$ antiferromagnetic correlations ( $d$-wave pair-field correlations) will dominate at low temperatures. We note that in the large $U / t$ limit, $T_{J}$ should scale as $t^{2} / U$. That up to $U / t=8$ we still do not see this behavior, may be traced back to the fact that the $W$-term enhances the band-width [15]. $T_{p}^{d} \equiv T_{J}$ is a natural consequence of the assumption of $S O(5)$ symmetry which unifies antiferromagnetism with $d$-wave superconductivity [16]. The data, however, does not demonstrate the presence of this symmetry in this model and further work is required.

In conclusion, we have considered aspects of the $t-$ $U-W$ model defined in Eq.(11). The model, at halfband filling, shows rich physics and allows us to study the interplay between magnetism and $d$-wave superconductivity. We have pointed out the surprising nature of the spin-spin correlations in the superconducting state: $S(\vec{r}) \sim e^{-i \vec{r} \cdot \vec{Q}}|\vec{r}|^{-\alpha}$, where $\vec{Q}=(\pi, \pi), \alpha$ is in the range $1<\alpha<2$ and depends on the coupling constants $W$ and $U$. Those conclusions are based on large scale calculations for system sizes up to $24 \times 24$. We have equally shown that the energy scales at which $d$-wave pairing and antiferromagnetic fluctuations occur are identical. The further understanding of spin and charge dynamics, as well as the doping of the model remains for further studies.

\section{ACKNOWLEDGMENTS}

M. Imada, D.J. Scalapino and M. Muramatsu are thanked for many instructive conversations. The computations were carried out on the T3E of the HLRS, Stuttgart, as well as on the T90 and T3E of the HLRZ, Jülich.

[1] J.G. Bednorz and K.A. Müller, Z. Phys. B 64, 189, (1986).

[2] F.F. Assaad, M. Imada and D.J. Scalapino, Phys. Rev. Lett. 77, 4592, (1996). Phys. Rev. B, Phys. Rev. B 56, 15001, (1997).

[3] F.F. Assaad and M. Imada Phys. Rev. B. 58, (1998).

[4] G. Sugiyama and S.E. Koonin, Annals of Phys. 168 1, (1986).

[5] S. Sorella, S. Baroni, R. Car, And M. Parrinello, Europhys. Lett. 8, 663, (1989). S. Sorella, E. Tosatti, S. Baroni, R. Car, and M. Parinello, Int. J. Mod. Phys. B1, 993, (1989).

[6] J.E.Hirsch, Phys. Rev. B 31, 4403, (1985).

[7] S.R. White et al. Phys. Rev. B40, 506, (1989).

[8] W. Kohn, Phys. Rev. 133A, 171, (1964).

[9] N. Byers and C.N. Yang, Phys Rev. Let.7, 46, (1961).
[10] C.N. Yang, Reviews of Mod. Phys. 34, 694 (1962).

[11] F.F. Assaad, W. Hanke and D.J. Scalapino, Phys. Rev. Lett. 71, 1915 (1993), Phys. Rev. B 49, 4327 (1994).

[12] Flux quantization, measures directly the superfluid density which does not require the knowledge of the symmetry of pairing correlations, the form factor of the Cooper pair, or the nature of the quasiparticle which constitute the Cooper pair. We note however that we not yet established firmly the occurrence of a flux minima at $\Phi / \Phi_{0}=0$ [2].

[13] At $U / t=2$ and $U / t=1$, one may show numerically that the vertex contribution to the $d$-wave pairing susceptibility grows quicker than the vertex contribution to the staggered spin susceptibility as a function of system size [15.

[14] We note that obtaining the exponent $\alpha$ from the size scaling of $S(\vec{Q})$ requires larger lattice sizes than the ones considered here. Short range spin-spin correlations included in $S(\vec{Q})$ are still important for the considered lattice sizes.

[15] F. F. Assaad et al. unpublished.

[16] S.C. Zhang, Science, 275, 1089, (1997).

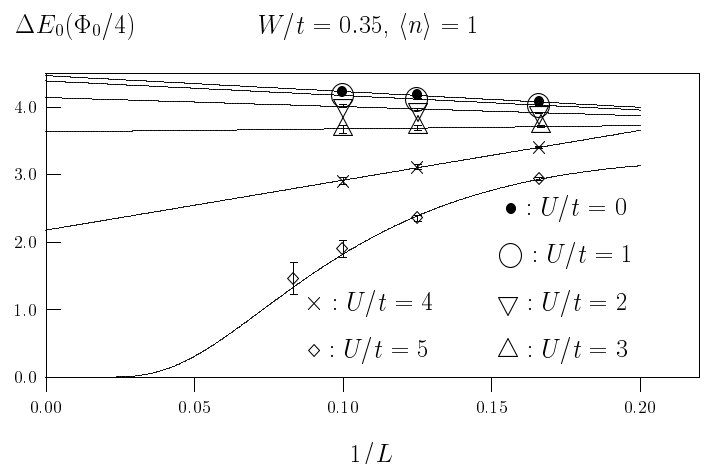

FIG. 1. $\Delta E_{0}\left(\Phi_{0} / 4\right) \equiv E_{0}\left(\Phi_{0} / 4\right)-E_{0}\left(\Phi_{0} / 2\right)$ as a function of inverse linear size $L$. For $U / t \leq 4$ the data is a consistent with a $1 / L$ form which extrapolates to a non-vanishing value in the thermodynamic limit. At $U / t=5$ the data may be fitted the SDW from $L \exp (-L / \xi)$ thus signaling an insulating state.

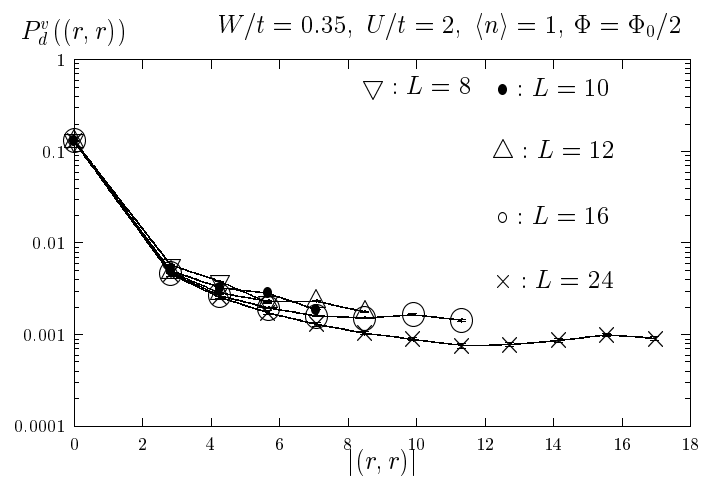

FIG. 2. Vertex contribution to the $d$-wave pair field correlations. Here, the temperature is set to $T=0$. 


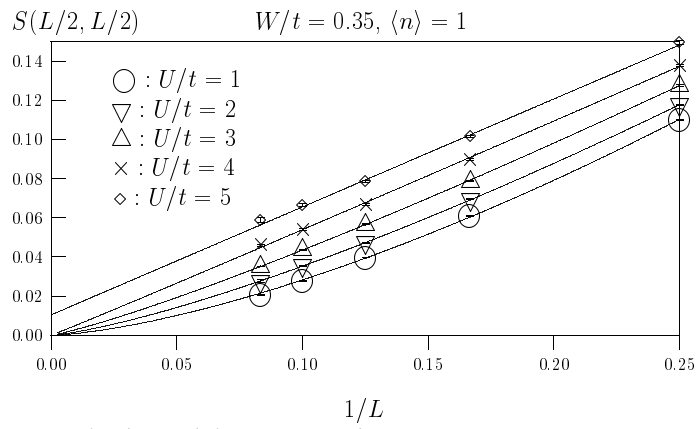

FIG. 3. $S(L / 2, L / 2)$ versus $1 / L$. The simulations were carried out at $\Phi / \Phi_{0}=0$. Here the $\Delta \tau$ systematic error is extrapolated to zero and the temperature is set to $T=0$. The solid lines are least square fits to the form: $L^{-\alpha}$. The result of the fit is summarized in Eq. (8).

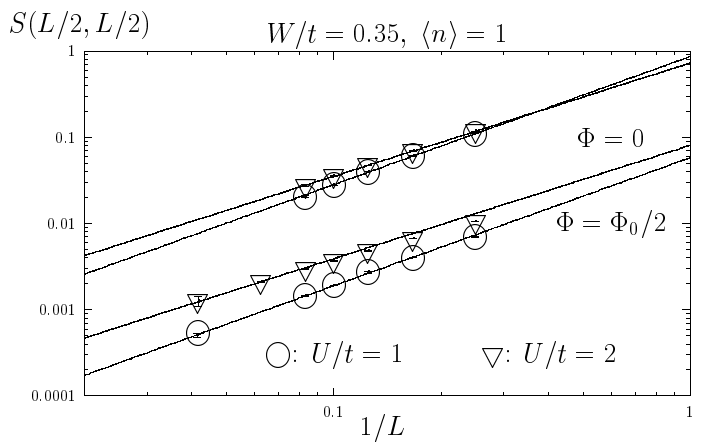

FIG. 4. $S(L / 2, L / 2)$ versus $1 / L$ for different boundary conditions. The solid line for the $\Phi=0$ data is obtained from least square fit to the form $L^{-\alpha}$. The same value of the exponent $\alpha$ was used to fit the data at $\Phi=\Phi_{0} / 2$. Here the $\Delta \tau$ systematic error is extrapolated to zero, and $T=0$.

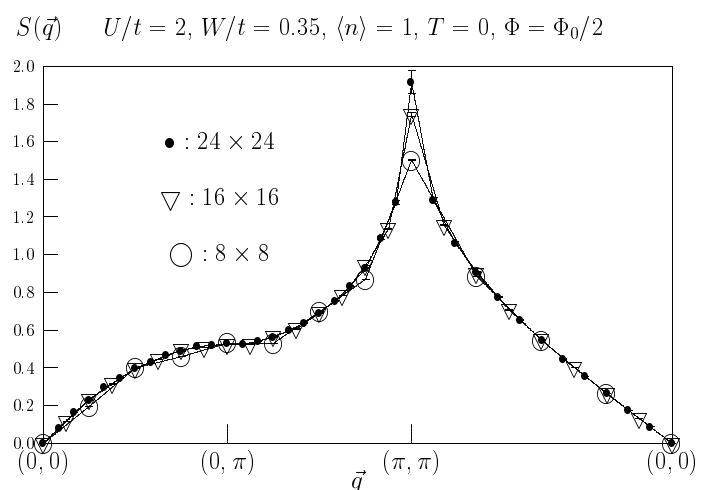

FIG. 5. Fourier transform of the equal time spin-spin correlations.

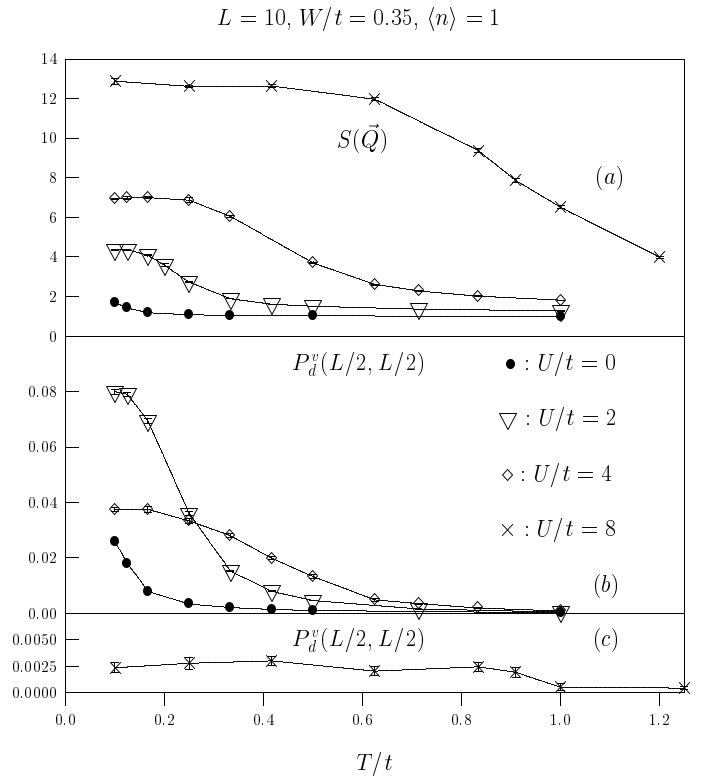

FIG. 6. (a) $S(\vec{Q}=(\pi, \pi))$ as a function of temperature, for different values of $U / t$. (b)-(c): $P_{d}^{v}(L / 2, L / 2)$ versus temperature. The calculations presented in the figure were carried out at $\Phi=0$.

\begin{tabular}{|c|c|c|}
\hline \hline$\vec{r}$ & $P_{s}^{v}(\vec{r})$ & $P_{d}^{v}(\vec{r})$ \\
\hline$(0,0)$ & $0.2950 \pm 0.0018$ & $0.1304 \pm 0.0011$ \\
$(0,1)$ & $0.0932 \pm 0.0009$ & $0.0238 \pm 0.0006$ \\
$(0,2)$ & $0.0076 \pm 0.0002$ & $0.0252 \pm 0.0003$ \\
\hline
\end{tabular}

TABLE I. Short range vertex contribution of pair-field correlations in the extended $s$ - and $d$-wave channels. Here we consider an $L=24$ lattice at $W / t=0.35, U / t=2$ and $\langle n\rangle=1$. The boundary conditions are set by $\Phi=\Phi_{0} / 2$. The distance $\vec{r}$ is in units of the lattice constant. 